\title{
Kilohertz high-frequency alternating current blocks nerve conduction without causing nerve damage in rats
}

\author{
Dandan Ling", Junjie Luo", Mengying Wang, Xiaodan Cao, Xiaorui Chen, Kexin Fang, Bin Yu \\ Department of Anesthesiology, Tongji Hospital Affiliated to Tongji University, Tongji University School of Medicine, Shanghai 200065, China \\ Contributions: (I) Conception and design: B Yu, D Ling; (II) Administrative support: Bin Yu; (III) Provision of study materials or patients: \\ D Ling, J Luo, M Wang, X Cao, X Chen, K Fang; (IV) Collection and assembly of data: M Wang, X Cao, X Chen, K Fang; (V) Data analysis and \\ interpretation: D Ling, J Luo; (VI) Manuscript writing: All authors; (VII) Final approval of manuscript: All authors. \\ \#These authors contributed equally to this work. \\ Correspondence to: Bin Yu. Doctor of Surgery of Clinical Medicine, Department of Anesthesiology, Tongji Hospital Affiliated to Tongji University, \\ Tongji University School of Medicine, No. 389 Xincun Road, Putuo District, Shanghai 200065, China. Email: yubin@tongji.edu.cn.
}

\begin{abstract}
Background: In recent years, $2-50 \mathrm{kHz}$ high-frequency alternating current has been shown to block nerve conduction mostly based on simulation models or experiments in vitro. This study aimed to assess the nerve block effects and related parameters of kilohertz alternating current in a rat model.

Methods: High-frequency biphasic rectangular stimulus pulse was applied to rat's sciatic nerve in vivo, and its blockade frequency and intensity was studied by recording the changes of compound muscle action potential (CMAP) amplitude and muscle states before and after stimulation. Secondly, diameter and circumference of sciatic nerve was measured at stimulating point by ultrasound. The correlation between stimulus' frequency and the nerve's diameter and circumference was studied. Lastly, we assessed nerve damage causing by high-frequency electrical stimulation by measuring CMAP and nerve conduction velocity (NCV) in the following day and sciatic nerve hematoxylin-eosin staining, both blocked side and contralateral side.

Results: When the current intensity was fixed, the blockade only occurred in a specific frequency range, above or below might have partial block effect. Preliminary statistical results showed that the blocking frequency of high-frequency alternating current was negatively linearly correlated with the circumference of sciatic nerve $(\mathrm{P}<0.05)$; HE staining of the sciatic nerve showed no axon and myelin sheath damage on blocked or opposite side, and the CMAP and NCV of the sciatic nerve remeasured in the next day were normal, indicating high-frequency electrical stimulation produced no nerve injury.
\end{abstract}

Conclusions: High-frequency alternating current stimulation can block nerve conduction without causing nerve damage, and the complete block frequency is negatively linearly correlated with the circumference of sciatic nerve.

Keywords: Nerve block; kilohertz frequency nerve block; high frequency; electrical stimulation; nerve conduction

Submitted Jun 27, 2019. Accepted for publication Sep 29, 2019.

doi: $10.21037 /$ atm.2019.10.36

View this article at: http://dx.doi.org/10.21037/atm.2019.10.36

\section{Introductions}

Nerve block anesthesia has advantages of good local analgesic effect, simply operation and few adverse reactions (1). However, it's now mainly achieved by local anesthetics. In clinical, multiple nerves block are commonly carried out, and with the deep insight into local anesthetics, the nerve injury (2), toxicity reaction (3), cell growth inhibition and apoptosis (4) resulted from megadose of local anesthetics have arouse investigators' attention. To date, many scholars have made efforts on nerve block localization technology, local anesthetic dosage and delivery form management, and toxicity mechanism, but all failed to solve this issue fundamentally. We wonder that whether it is possible to 


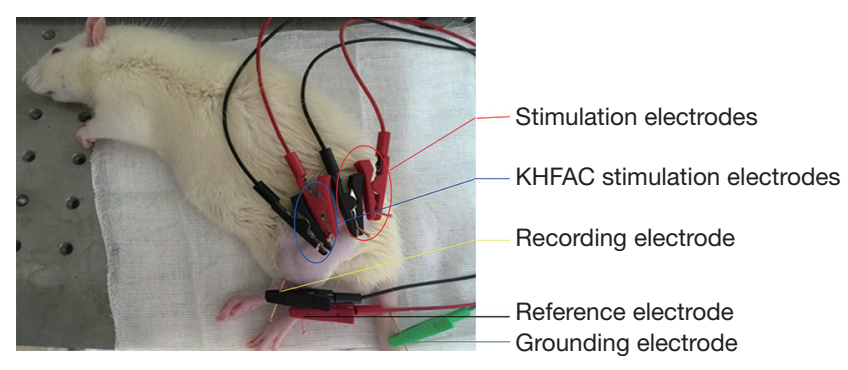

Figure 1 Electrodes' location on the rat.

block peripheral nerve conduction by physical method (such as electrical stimulation) considering the nature of nerve conduction — the conduction of action potential.

Previous studies have documented that, electric stimulation can block nerve conduction under certain condition, in addition to generating action potentials. Compared to chemical block, physical electric stimulation technique has incomparable advantages in terms of immediacy, mini-invasive, selectivity and controlling (5). Kilohertz high-frequency alternating current (KHFAC) refers to charge-balanced alternating current with frequency ranging from 1 to $100 \mathrm{kHz}$. Both amphibian and mammalian axon models researches indicated that kilohertz high-frequency biphasic rectangular current pulse can achieve axon conduction block in the range of 4 to $10 \mathrm{kHz}$ (6). Due to potential clinical applications, many experiments have been performed to investigate the blocking phenomenon and mechanisms and most are computer axonal model stimulation or in vitro axon model (7). It's difficult to investigate KHFAC stimulation in vivo because of the unknown mechanism and unsafely. At present, the unclear issue is whether KHFAC can block nerve conduction in vivo, and whether such stimulation will cause nerve damage. In addition, at a certain current intensity, the relationship between the blocking frequency and the nerve also needs to be further studied. So we administered this study to test KHFAC's blocking effect and related parameters in a rat model.

\section{Methods}

\section{Experimental animal}

Sprague Dawley (SD) male rats weighing 200-450 g were purchased from the Shanghai Slac Laboratory Animal Co. Ltd. [license No. SCXK (Shanghai)2013-0006]. The animals are housed in the SPF (specific perception free) animal houses with 12-hour circadian cycles and free access to food and water. All experiments were conducted in accordance with the ethical regulations of experimental animals in Shanghai Tongji Hospital.

\section{Sciatic nerve CMAP measurement}

The nerve evoked potential equipment and electrophysiological monitoring equipment (Nuocheng Co., China) were adopted to induce and record the sciatic nerve's CMAP. Acupuncture needle $(0.3 \mathrm{~mm} \times 13 \mathrm{~mm}$, Hwato Co., China) was used as electrode to conduct electrical signal.

After narcotized by $2 \%$ pentobarbital sodium $(30 \mathrm{mg} / \mathrm{kg}$, rat's mass), the rat was fixed on a plate in a lateral position. The posterior lateral area of the thigh and the anterior side of the calf were shaved and disinfected. The anode and cathode of stimulation electrodes were placed near to great trochanter of femur. The recording electrode and reference electrode were placed in the tibial anterior muscle belly and tendon, respectively. A grounding electrode was placed in the rat tail (Figure 1).

Parameters of evoked potential equipment were set as: frequency $2 \mathrm{~Hz}$, wave width $0.1 \mathrm{~ms}$, band-pass frequency $2-10 \mathrm{kHz}$, stimulus intensity increased by $30 \%$ compared with threshold intensity.

\section{Sciatic nerve's diameter and circumference measurement with ultrasound guidance}

After anesthesia and depilation, we used a L18-4 linear array probe (frequency range 4-18 MHz) (Sonimage HS1, Konica Minolta Co., China) to measure the diameter and circumference of nerve and to locate the stimulation electrode. The detection depth of the screen was $1 \mathrm{~cm}$. The sciatic nerve of rat is mainly composed of the lumbar spinal nerve (L4-L6), after travelling in front of the sacroiliac joint and bypassing the great trochanter of the femur and reaching the rear of the femur, the nerve finally divides into three major branches, namely the common peroneal nerve, tibial nerve and sural nerve.

Anatomical measurement data showed that the femur length of SD rats was about $3 \mathrm{~cm}$, the length of the posterior femur segment of the sciatic nerve trunk was about $1.7 \mathrm{~cm}$, and the midpoint of the femur was about $0.5 \mathrm{~cm}$ from the upper margin of the sciatic nerve trunk, that is, the beginning of the branch of the sciatic nerve trunk was below the midpoint of the femur. So the probe was placed in the greater trochanter of the femur and moved downwards. 


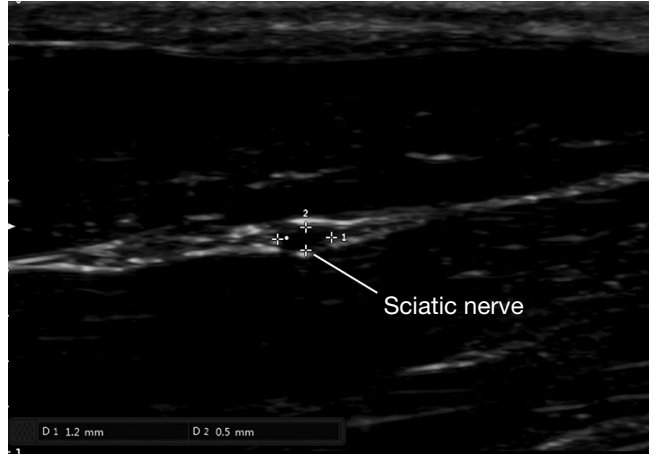

Figure 2 The sciatic nerve was located under ultrasound and its diameter was measured.

Below the midpoint of the femur, the probe was placed perpendicular to the femur to measure the diameter and circumference of the sciatic nerve (Figure 2).

\section{KHFAC blocks the nerve conduction}

The stimulation point was located at the distal end of the sciatic nerve and below the midpoint of the femur. The location was determined by measuring the sciatic nerve CMAP and ultrasound guidance. If CMAP of sciatic nerve could be recorded when the stimulation current intensity was less than $1 \mathrm{~mA}$, it indicated that the stimulation point was near the sciatic nerve.

High-frequency stimulation signal parameters were set as follow: frequency was 4 to $20 \mathrm{kHz}$, gradient was $1 \mathrm{kHz}$; Current intensity rang was 1 to $10 \mathrm{~mA}$; Time control was stimulation time $10 \mathrm{~s}$ with rest $5 \mathrm{~s}$, total time reached $30 \mathrm{~min}$; stimulation pulse width was calculate according to formula: pulse width $=[(1 /$ frequency $) \times 0.8] / 2 \times 10^{6}$, the occupation ratio was 0.8 . Stimulus waveform was biphasic rectangular wave.

\section{Statistical analyses}

Spearman correlation rank sum test and chi-square fitting test were used to evaluate correlation between stimulation's frequency and intensity and the nerve's diameter and circumference. $\mathrm{P}<0.05$ means statistically significant.

\section{Results}

\section{KHFAC stimulation can block sciatic nerve conduction in vivo}

The result showed that the high-frequency electric stimulation can block the conduction of sciatic nerve in vivo. When the intensity maintained to a certain value, the stimulation had block effect with a range of frequency, and the stimulation might have no block effect above or below this frequency rang (Figure 3).

\section{At a certain current intensity, the blocking frequency is correlated with the nerve circumference}

We applied Spearman correlation rank sum test and chisquare fitting test to our date. In Table 1, the first column exhibited the linear model, logarithm model, quadratic model and cubic exponential model respectively. It was considered to be consistent when the sig. (P value) $<0.05$. All the models in this example could be fitted. At that time, the one with a larger R Square (indicating better fitting effect) could be selected. If the situation were similar, a simpler model would be recommended. In this case, we preferred to a linear model, the quadratic model with a largest $\mathrm{R}$ square was considerable according to the actual

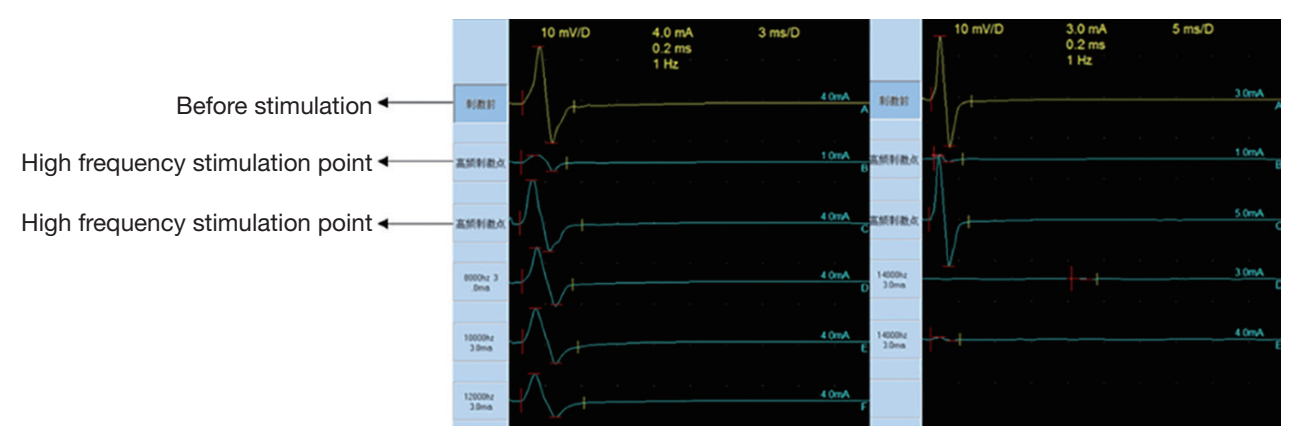

Figure 3 The electromyography results with high frequency stimulation. For example, when the current intensity reached $3 \mathrm{~mA}, 14 \mathrm{kHz}$ stimulation had total block effect while 8,10 and $12 \mathrm{kHz}$ failed to block the nerve conduction. 
Table 1 Model summary and parameter estimates

\begin{tabular}{|c|c|c|c|c|c|c|c|c|c|}
\hline Equation & \multicolumn{5}{|c|}{ Model summary } & \multicolumn{4}{|c|}{ Parameter estimates } \\
\hline Linear & 0.327 & 7.275 & 1 & 15 & 0.017 & $21,759.282$ & $-3,027.673$ & & \\
\hline Logarithmic & 0.292 & 6.188 & 1 & 15 & 0.025 & $22,026.027$ & $-8,778.508$ & & \\
\hline Quadratic & 0.370 & 4.116 & 2 & 14 & 0.039 & $7,145.121$ & $6,497.639$ & $-1,484.737$ & \\
\hline Exponential & 0.273 & 5.638 & 1 & 15 & 0.031 & $33,180.243$ & -0.331 & & \\
\hline
\end{tabular}

The independent variable is nerve circumference. Dependent variable: stimulation frequency.

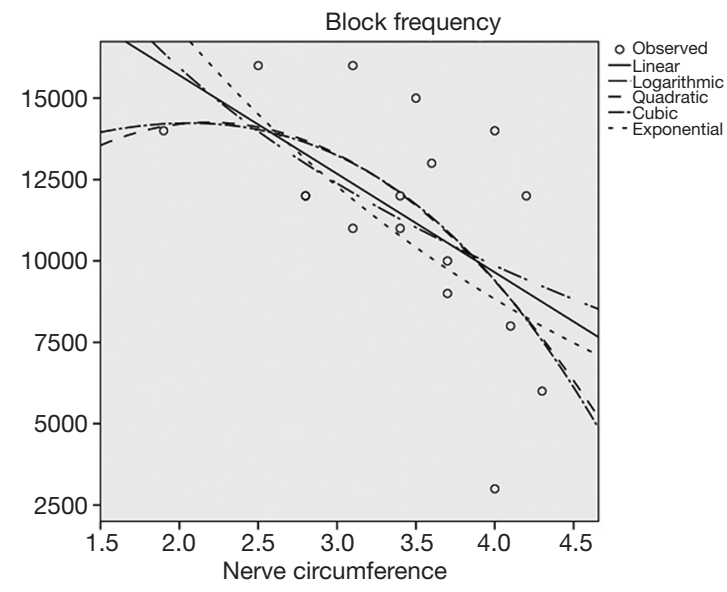

Figure 4 Block frequency had a negative linear correlation with the sciatic nerve's circumference.

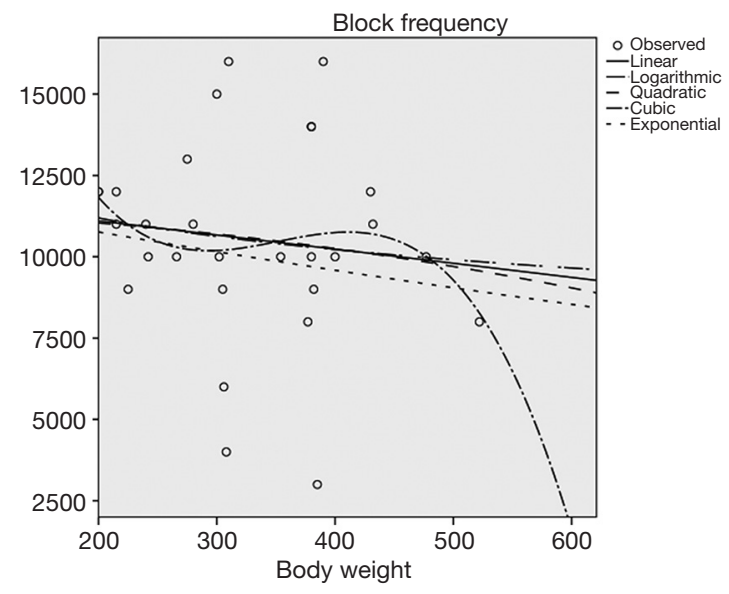

Figure 5 There was no correlation between the block frequency and the rat's body weight.

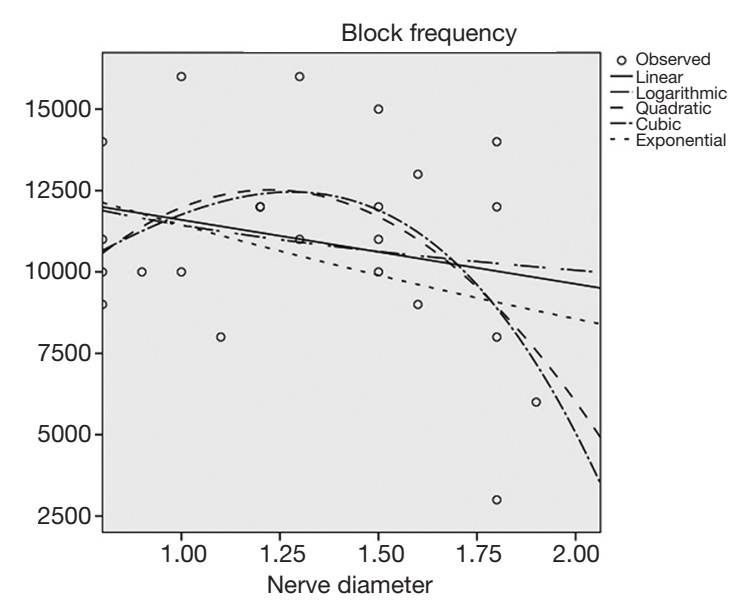

Figure 6 There was no correlation between the block frequency and the rat's nerve diameter.

situation (Table 1).

According to the preliminary results of the experiment, there was a negative linear correlation between the block frequency and the circumference of the sciatic nerve $(\mathrm{P}<0.05)$ (Figure 4), and no correlation between the block frequency and the nerve's transverse diameter and body weight $(\mathrm{P}>0.5)$ (Figures 5 and 6 ).

\section{Under a certain current intensity, KHFAC completely blocks sciatic nerve conduction without causing nerve damage}

We observed lost motor function in rat's blocking limb after 30 minutes of high-frequency electrical stimulation. CMAP amplitude of the sciatic nerve and motor function were fully 


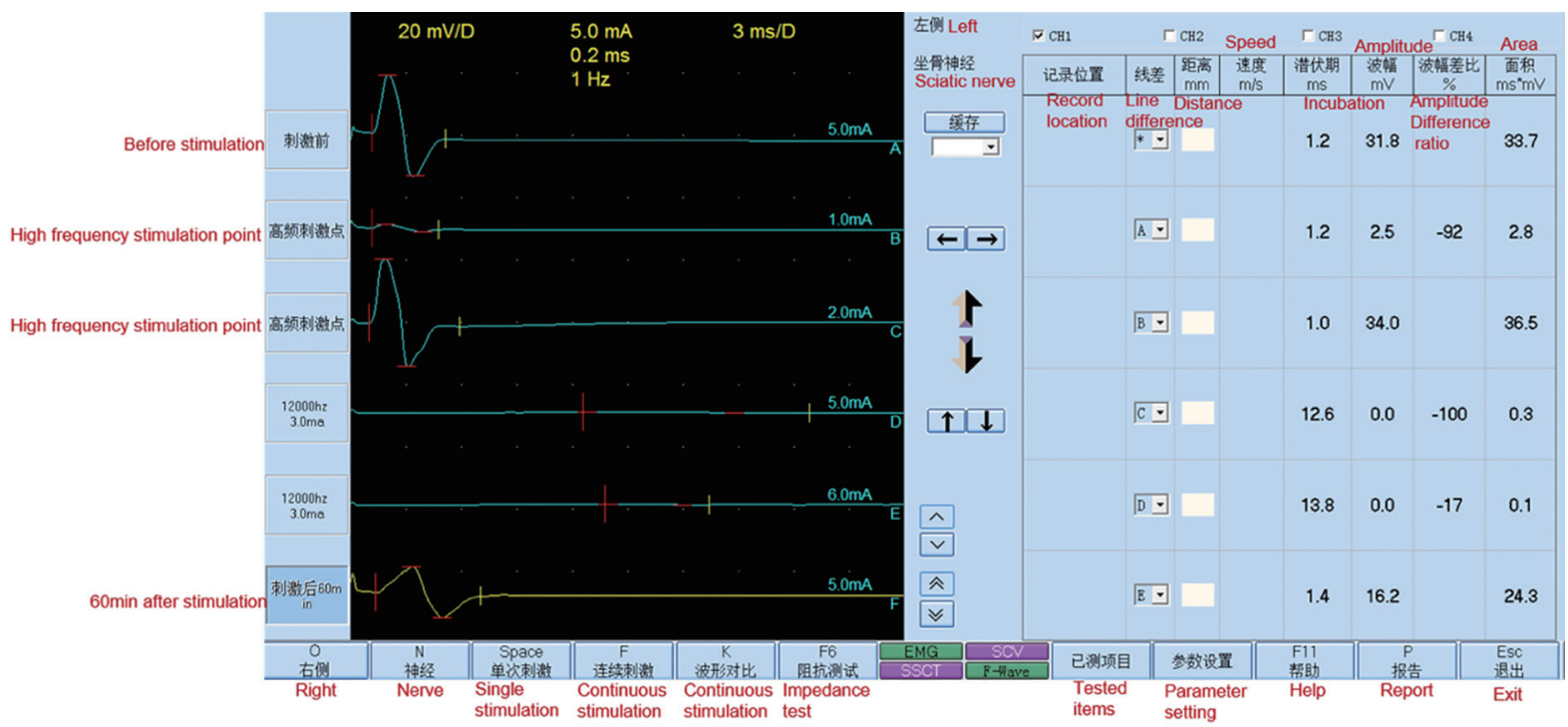

Figure 7 The sciatic nerve was total blocked when the current intensity reached $3 \mathrm{~mA}, 12 \mathrm{kHz}$ stimulation. And the CMAP amplitude of the sciatic nerve and motor function were fully restored to pre-block level after 60 minutes recovery. CMAP, compound muscle action potential.

restored to pre-block level after 40 to 60 minutes recovery (Figure 7). After the block, HE staining was performed on the stimulated and contralateral sciatic nerve tissues, showing no axon and myelin sheath damage (Figure 8). The CMAP and nerve conduction velocity $(\mathrm{NCV})$ of the sciatic nerve remeasured in the following day were normal, indicating that the high frequency electrical stimulation did not cause nerve damage.

\section{Discussion}

The implementation of present nerve block technique mostly depends on the application of local anesthetics, which almost all have neurotoxicity, even at low concentrations (8). In addition, the increasing reports of neurological complications have drawn much clinical attention. Therefore, it is meaningful to find a suitable way to physically block nerve conduction.

Previous studies on high-frequency electrical stimulation mainly relied on establishing in vitro simulation model, such as Hodgkin-Huxley (H-H) (9) model and FrankenhaeuserHuxley (F-H) (10) model. Amphibians' sciatic nerve was commonly adopted for determination of NCV in such in vitro experiments. Amphibians' nervous system development is far less than one in mammals. Among all kinds of experimental animals, rats have a complete strain, which can provide cheap mammalian nerve tissue. The structure of rats' sciatic nerve tissue is not significantly different from that of human peripheral nerve, even under the electron microscope (11). Therefore, in this study, we chose SD rats' sciatic nerve as experimental subject, and high-frequency electric stimulation was applied to blocking the conduction of the sciatic nerve in vivo via minimally invasive method. It has great clinical application and reference value as it is similar to the natural physiological condition with little trauma.

Nerve conduction detection (NCS) is defined as the recording of impulses from the site where the action potential is induced by a peripheral nerve to some parts of the distal part of the nerve. In other words, it stimulates the nerve along one or more sites and records the electrical activity of the nerve. The nerve injury can be evaluated by examining the ability of nerve conduction impulse. The most common nerve conduction tests included motor nerve compound muscle action potentials (CMAP), sensory nerve action potentials (SNAP), mixed (sensory and motor) nerve action potentials [compound nerve action potentials (CNAP)] and delayed response (mainly $\mathrm{F}$-wave and $\mathrm{H}$ reflex). This in vivo study aimed to assess the nerve blocking effects and related parameters of kilohertz alternating current in rats, by recording the changes of CMAP amplitude and muscle states before and after HF stimulation, and whether HF stimulation induces any nerve damage. 

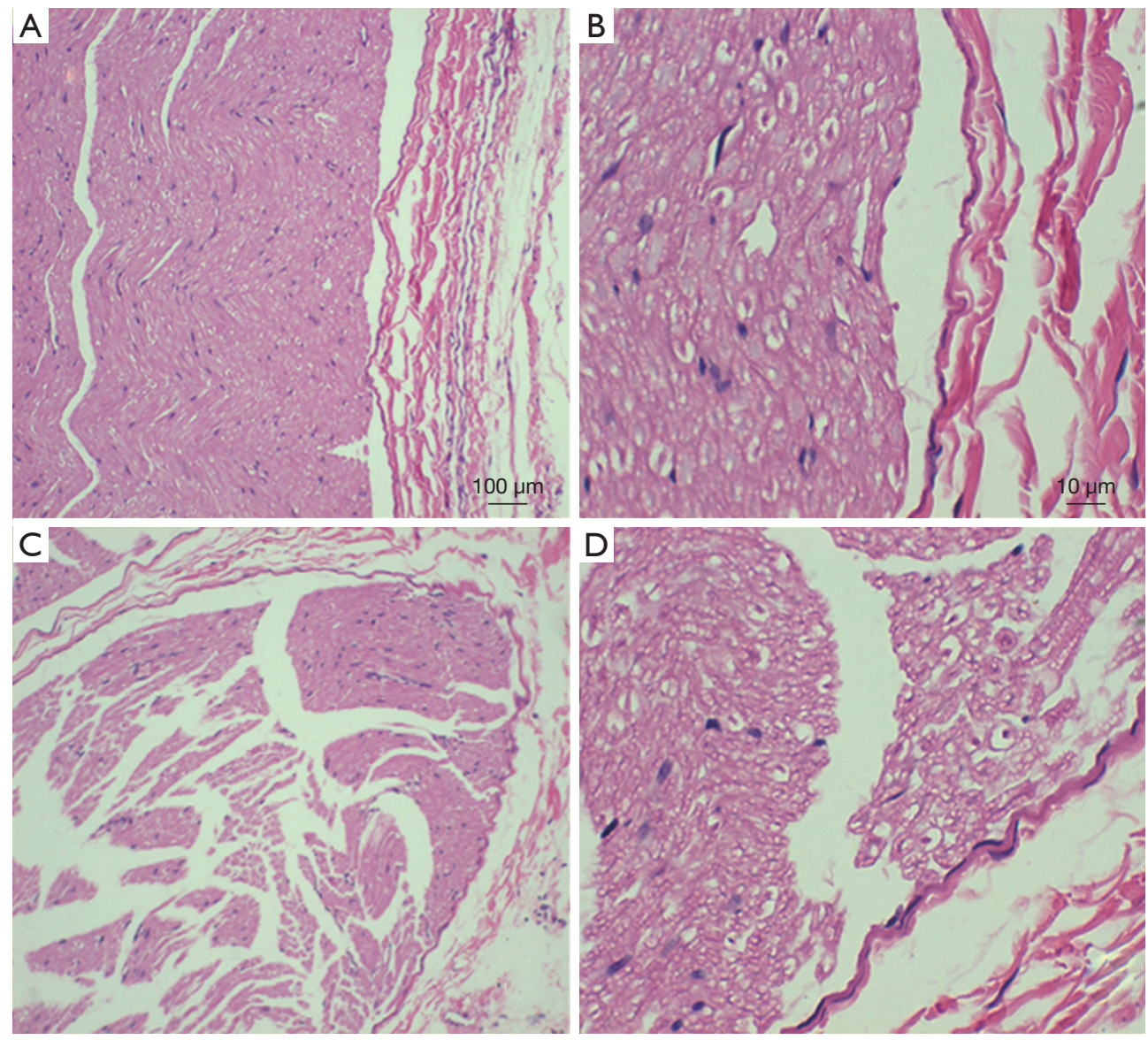

Figure 8 The hematoxylin-eosin (HE) staining of nerve tissue. Control group: (A, ×40; B, ×400), experimental group: (C, ×40; D, ×400). The neuronal cells were star-, triangle- and spindle-shaped, with clear cell body and nucleus. There was no significant changes of cell swelling, nuclear lysis or other acute injury. There was no significant change in nerve fiber density, axonal damage such as axonal fracture, disintegration disappearance and myelin sheath disintegration disappearance (Waller degeneration). No changes of chronic injury such as neuronal atrophy and glial cell proliferation were observed.

At a certain frequency, the minimum blocking intensity that completely attenuates the action potential amplitude of neural trunk to 0 is defined as the blocking threshold (12), at which the neural action potential is considered to be completely blocked. In this experiment, we found that when the blocking intensity maintained a certain value, the blocking only occurred in a frequency range, and no blocking effect could be achieved in the lower or higher frequency bounds. When the stimulation intensity did not reach the blocking threshold, the muscle was always in tetanic contraction when the high-frequency stimulation signal was applied. But the muscle state was a continuously changed process when the high-frequency stimulation signal was applied with a threshold intensity, beginning as a tetanic contraction lasting for several milliseconds (onset response) $(5,13)$, and then slowly relaxing until the next stimulation. Onset response is one of the reasons affecting the wide application of KHFAC stimulation in clinical practice. Currently, Onset response can be reduced or even eliminated through direct current (DC) combined KHFAC $(14,15)$, but the safety of its long-term application is not clear, which is also what we should study in the following experiments.

The total stimulation time in this experiment was $60 \mathrm{~min}$. After the blockade, CMAP of the sciatic nerve was measured intermittently, and it was found that the amplitude of the action potential gradually increased and returned to the pro-block level about $1 \mathrm{~h}$ later. However, Kilgore $e t a l$. believe that the recovery and delay effects of KHFAC to block neural conduction function can be divided 
into three different types according to the length of time of applied blocking stimulus. Taking the recovery of muscle tension as the measurement standard, when the stimulation time is less than $15 \mathrm{~min}$, the muscle tension can be immediately restored within $3 \mathrm{~s}$, namely the instantaneous recovery. When the stimulation lasts 15 to $40 \mathrm{~min}$, the muscle tension can be completely restored within $3 \mathrm{~min}$, that is, the rapid recovery. However, when the stimulation time is longer than $40 \mathrm{~min}$, the complete recovery of muscle tension takes several hours, that is, the slow recovery. This effect is called the "carry over" effect (5). In this experiment, the block time was $60 \mathrm{~min}$, and the histological observation of the homolateral and contralateral sciatic nerve and the electromyography detection of the sciatic nerve restoring the block showed no nerve damage. Of course, whether the nerve damage was caused with the extension of the stimulation time still needs to be further studied.

Although high-frequency electrical stimulation nerve conduction's blocking effect on somatic and autonomic nerve in mammals $(16,17)$ and non-mammals $(7)$ model are confirmed, but the way to realize the safe, effective and reversible nerve conduction block is influenced by the electrode, and existing studies emphasize the role of the polar distance (13) and electrode geometry (18). This experiment chooses the acupuncture needle as stimulating electrode, and the whole tip conducts electricity. Due to the low spatial resolution caused by the current diffusion and the position deviation, there is a volume effect, and the current intensity needs to be increased to achieve the inhibitory effect. High intensity current causes a large amount of charge injection, which increases the risk of nerve damage. Therefore, it is necessary to study the conducting electrode at the tip to reduce the charge dispersion and improve the blocking success rate. In this experiment, the needle-level electrode was completely punctured into the muscle, and nearly no shift was generated in the highfrequency electrical stimulation process. If the needlelevel electrode was slightly displaced, the current could still generate the CMAP. If CMAP was disappeared while increasing the current, the nerve conduction block could be explained.

It is possible to selectively activate and inhibit different nerve fibers by electrical stimulation, but it is very important to select appropriate current intensity. The diameter of nerve fibers (19), the shape of electrodes (20), the contact mode between electrodes and nerves (21), the distance between electrodes (22) and other factors all affect the stimulation parameters such as the frequency and intensity of blocking signals, so it is very necessary to master the setting rules of electrical stimulation parameters.

This study only preliminarily determined the relationship between the diameter and circumference of the sciatic nerve in rats and the frequency of high-frequency electrical stimulation when the nerve conduction was completely blocked under a certain current intensity, and concluded that there was a linear negative correlation between the nerve circumference and the frequency of electrical stimulation. But due to the low sensitivity of ultrasonic measuring animal nerve diameter, the measured diameter and electrical stimulation parameters were not statistically significant. Since the number of experimental cases measured in this experiment was relatively small, further experiments are needed to expand the sample size to obtain a more accurate correlation between the two.

\section{Conclusions}

KHFAC stimulation can block nerve conduction without causing nerve damage, and the complete block frequency is negatively linearly correlated with the circumference of sciatic nerve.

\section{Acknowledgments}

Funding: This study was funded by the Science and Technology Commission Shanghai Municipality project (No. 18441905700) and Shanghai Hospital Development Center project (No. 16CR3004A).

\section{Footnote}

Conflicts of Interest: The authors have no conflicts of interest to declare.

Ethical Statement: The authors are accountable for all aspects of the work in ensuring that questions related to the accuracy or integrity of any part of the work are appropriately investigated and resolved. Sprague Dawley male rats were purchased from the Shanghai Slac Laboratory Animal Co. Ltd. [license No. SCXK (Shanghai)2013-0006]. All experiments were conducted in accordance with the ethical regulations of experimental 
animals in Shanghai Tongji Hospital.

\section{References}

1. Liu SS, Ngeow JE, Yadeau JT. Ultrasound-guided regional anesthesia and analgesia: a qualitative systematic review. Reg Anesth Pain Med 2009;34:47-59.

2. Shah S, Hadzic A, Vloka JD, et al. Neurologic complication after anterior sciatic nerve block. Anesth Analg 2005;100:1515-7, table of contents.

3. Klein SM, Pierce T, Rubin Y, et al. Successful resuscitation after ropivacaine-induced ventricular fibrillation. Anesth Analg 2003;97:901-3.

4. Maurice JM, Gan Y, Ma FX, et al. Bupivacaine causes cytotoxicity in mouse C2C12 myoblast cells: involvement of ERK and Akt signaling pathways. Acta Pharmacol Sin 2010;31:493-500.

5. Kilgore KL, Bhadra N. Reversible nerve conduction block using kilohertz frequency alternating current. Neuromodulation 2014;17:242-54; discussion 254-5.

6. Liu H, Roppolo JR, de Groat WC, et al. The role of slow potassium current in nerve conduction block induced by high-frequency biphasic electrical current. IEEE Trans Biomed Eng 2009;56:137-46.

7. Joseph L, Butera RJ. High-frequency stimulation selectively blocks different types of fibers in frog sciatic nerve. IEEE Trans Neural Syst Rehabil Eng 2011;19:550-7.

8. Kishimoto T, Bollen AW, Drasner K. Comparative spinal neurotoxicity of prilocaine and lidocaine. Anesthesiology 2002;97:1250-3.

9. Hodgkin AL, Huxley AF. A quantitative description of membrane current and its application to conduction and excitation in nerve. J Physiol 1952;117:500-44.

10. Frankenhaeuser B, Huxley AF. The action potential in the myelinated nerve fiber of Xenopus laevis as computed on the basis of voltage clamp data. J Physiol 1964;171:302-15.

11. Mackinnon SE, Hudson AR, Hunter DA. Histologic assessment of nerve regeneration in the rat. Plast Reconstr Surg 1985;75:384-8.

Cite this article as: Ling D, Luo J, Wang M, Cao X, Chen X, Fang K, Yu B. Kilohertz high-frequency alternating current blocks nerve conduction without causing nerve damage in rats. Ann Transl Med 2019;7(22):661. doi: 10.21037/atm.2019.10.36
12. Bhadra N, Kilgore KL. High-frequency electrical conduction block of mammalian peripheral motor nerve. Muscle Nerve 2005;32:782-90.

13. Ackermann DM Jr, Bhadra N, Foldes EL, et al. Effect of nerve cuff electrode geometry on onset response firing in high-frequency nerve conduction block. IEEE Trans Neural Syst Rehabil Eng 2010;18:658-65.

14. Ackermann DM Jr, Bhadra N, Foldes EL, et al. Conduction block of whole nerve without onset firing using combined high frequency and direct current. Med Biol Eng Comput 2011;49:241-51.

15. Franke M, Vrabec T, Wainright J, et al. Combined KHFAC + DC nerve block without onset or reduced nerve conductivity after block. J Neural Eng 2014;11:056012.

16. Patel YA, Saxena T, Bellamkonda RV, et al. Kilohertz frequency nerve block enhances anti-inflammatory effects of vagus nerve stimulation. Sci Rep 2017;7:39810.

17. Patel YA, Butera RJ. Differential fiber-specific block of nerve conduction in mammalian peripheral nerves using kilohertz electrical stimulation. J Neurophysiol 2015;113:3923-9.

18. Patel YA, Kim BS, Rountree WS, et al. Kilohertz Electrical Stimulation Nerve Conduction Block: Effects of Electrode Surface Area. IEEE Trans Neural Syst Rehabil Eng 2017;25:1906-16.

19. Jensen AL, Durand DM. High frequency stimulation can block axonal conduction. Exp Neurol 2009;220:57-70.

20. Ackermann DM Jr, Foldes EL, Bhadra N, et al. Effect of bipolar cuff electrode design on block thresholds in highfrequency electrical neural conduction block. IEEE Trans Neural Syst Rehabil Eng 2009;17:469-77.

21. Ackermann DM Jr, Foldes EL, Bhadra N, et al. Conduction block of peripheral nerve using highfrequency alternating currents delivered through an intrafascicular electrode. Muscle Nerve 2010;41:117-9.

22. Tai C, de Groat WC, Roppolo JR. Simulation of nerve block by high-frequency sinusoidal electrical current based on the Hodgkin-Huxley model. IEEE Trans Neural Syst Rehabil Eng 2005;13:415-22. 